\title{
Perfil sociodemográfico e fatores de atração e saída dos médicos atuantes na estratégia saúde da família no município de Ponta Grossa, Paraná, Brasil
}

\author{
Sociodemographic profile and factors of attraction and exit of doctors in the family health strategy \\ in Ponta Grossa, Paraná, Brazil \\ Perfil sociodemográfico y factores de atracción y salida de los médicos que actuan en la \\ estrategia de salud familiar en Ponta Grossa, Paraná, Brasil
}

\author{
Lorena Slusarz Nogueira $^{1 \oplus}$, Manoelito Ferreira Silva Junior ${ }^{\circledR}$, Erildo Vicente Müller $^{1 \oplus}$ \\ ${ }^{1}$ Universidade Estadual de Ponta Grossa (UEPG), Ponta Grossa, PR
}

\section{Resumo}

Introdução: Diversos estudos destacam a alta rotatividade e a dificuldade de fixação dos profissionais nas equipes, especialmente médicos, como um dos grandes desafios para a consolidação da estratégia saúde da família (ESF) no Brasil. A rotatividade prejudica a longitudinalidade do cuidado, a formação do vínculo com a equipe e a qualidade da assistência prestada. Objetivo: Descrever o perfil sociodemográfico e os fatores de atração e saída de médicos inseridos na ESF. Métodos: Estudo transversal com abordagem quantitativa, realizado entre março e abril de 2019, do município de Ponta Grossa-PR, Brasil, por meio de questionário semiestruturado e autoaplicado desenvolvido no estudo. A análise descritiva foi realizada por frequências relativas (\%) e absolutas (n). Resultados: Participaram 61 médicos. Houve predomínio de profissionais mulheres $(57,4 \%)$, com menos de 30 anos (49,2\%), formou-se após $2015(60,7 \%)$ e não é natural do município (82\%). Apenas 1,6\% possuía residência em medicina de família e comunidade e $9,8 \%$ especialização em saúde da família. Foi alto o percentual de médicos contratados pelo "Programa Mais Médicos" (82\%) e que trabalha na ESF há menos de 6 meses (59\%). A identificação com o trabalho foi apontada como o principal fator que levou à inserção na ESF, enquanto o excesso de demanda e de processos burocráticos foi mencionado como importante fator de desmotivação. O principal motivo de saída dos profissionais foi a realização de residência médica. Conclusão: Houve predomínio de profissionais com baixo tempo de trabalho na ESF do município oriundos do "Programa Mais Médicos", em contratos temporários e relações trabalhistas precárias. A rotatividade médica é um assunto complexo, mas estratégias de valorização da carreira na atenção primária à saúde e a oferta de melhores condições de trabalho podem contribuir para sua resolução.

Palavras-chave: Atenção Primária à Saúde, Serviços de Saúde Comunitária, Estratégia Saúde da Família, Administração de Recursos Humanos.
Como citar: Nogueira LS, Silva Junior MF, Müller EV. Perfil sociodemográfico e fatores de atração e saída dos médicos atuantes na estratégia saúde da família no município de Ponta Grossa, Paraná, Brasil. Rev Bras Med Fam Comunidade. 2021;16(43):2159. https://doi.org/10.5712/rbmfc16(43)2159

\author{
Autor correspondente: \\ Lorena Slusarz Nogueira. \\ E-mail: Iorenaslu@ hotmail.com \\ Fonte de financiamento: \\ não se aplica. \\ Parecer CEP: \\ № 3.183.547. \\ Procedência: \\ não encomendado. \\ Avaliação por pares: \\ externa. \\ Recebido em: 03/08/2019. \\ Aprovado em: 05/07/2021.
}




\begin{abstract}
Introduction: Several studies have demonstrated the high turnover and the difficulty of fixing professionals in the work teams, especially doctors, as one of the great challenges for the consolidation of family health strategy (FHS) in Brazil. Professional turnover impairs the longitudinality of care, the formation of the bond with the team and the quality of care provided. Objectives: To describe the sociodemographic profile and the attraction and exit factors between doctors inserted in the family health strategy. Methods: The cross-sectional study with quantitative approach, carried out between March and April 2019, in the city of Ponta Grossa-PR, Brasil, through a semi-structured and self-applied questionnaire developed in the study. Descriptive analysis was performed by relative (\%) and absolute (n) frequencies. Results: 61 physicians participated. There was a predominance of female professionals $(57.4 \%)$, with less of 30 years-old $(49,2 \%)$, graduated after $2015(60,7 \%)$ and are not born in the city $(82 \%)$. Only $1.6 \%$ had residency in family and community medicine and $9.8 \%$ specialized in family health. It was high percentage of doctors hired by the "Programa Mais Médicos" (82\%) and who worked at the FHS for less than six months were found (59\%). The main reason for leaving the professionals is to perform medical residency. The identification with the work was pointed out as the main factor for the insertion in the ESF, while the excess of demand and bureaucratic processes was mentioned as an important demotivation factor. Conclusion: There was a predominance of professionals with a short working time in the municipality's PHS who came from the "Programa Mais Médicos", in temporary contracts and in precarious labor relations. Medical turnover is a complex issue, but strategies for career enhancement in primary care and the provision of better working conditions can contribute to its resolution.
\end{abstract}

Keywords: Primary Health Care, Community Health Services, Family Health Strategy, Personnel Management.

\title{
Resumen
}

Introducción: Varios estudios destacan la alta rotación y la dificultad de fijación de los profesionales en los equipos, especialmente médicos, como uno de los principales desafíos para la consolidación de la estrategia de salud familiar (ESF) en Brasil. La rotación perjudica la longitudinalidad de la atención, la formación de vínculos con el equipo y la calidad de la atención proporcionada. Objetivo: Describir el perfil sociodemográfico y los factores de atracción y salida de los médicos que laboran en la ESF. Método: Estudio transversal con enfoque cuantitativo, realizado entre marzo y abril de 2019, en la ciudad de Ponta Grossa-PR, Brasil, mediante un cuestionario semiestructurado y autoaplicado desarrollado en el estudio. El análisis descriptivo se realizó por frecuencias relativas (\%) y absolutas (n). Resultados: Participaron 61 médicos. Predominó el sexo femenino $(57,4 \%)$, menor de 30 años $(49,2 \%)$, se graduaron después de $2015(60,7 \%)$ y no son del municipio $(82 \%)$. Solo el $1,6 \%$ tenía residencia en medicina familiar y comunitaria y el $9,8 \%$ se especializó en salud de la familia. Fue alta porcentaje de médicos contratados por el "Programa Mais Médicos" (82\%) y que trabajan en la ESF por menos de 6 meses (59\%). La razón principal que conduce a la salida de profesionales es la realización de la residencia médica. La identificación con el trabajo se señaló como el factor principal que condujo a la inserción en el ESF, mientras que la demanda excesiva y los procesos burocráticos se mencionaron como un importante factor de desmotivación. Conclusión: Predominó la presencia de profesionales con poco tiempo de trabajo en la ESF del municipio, provenientes del "Programa Mais Médicos", en contratos temporales y en relaciones laborales precarias. La rotación médica es un tema complejo, pero las estrategias para mejorar la carrera en Atención Primaria y ofrecer mejores condiciones de trabajo pueden contribuir a su resolución.

Palabras clave: Atención Primaria de Salud, Servicios de Salud Comunitaria, Estrategia de Salud Familiar, Administración de Personal

\section{INTRODUÇÃO}

A atenção primária à saúde (APS) pode ser entendida como o primeiro nível do sistema de serviço de saúde, o qual deve funcionar como porta de entrada preferencial do sistema, com ações resolutivas sobre os problemas de saúde. Além disso, deve estar articulada com os demais níveis de complexidade, formando assim uma rede integrada de serviços. ${ }^{1}$ No Brasil, a estratégia saúde da família (ESF) é o modelo principal de implementação e organização da APS. ${ }^{2}$

A ESF é estruturada por equipes multidisciplinares, formadas, no mínimo, por médico, enfermeiro, técnico de enfermagem e agentes comunitários de saúde. ${ }^{2} \mathrm{~A}$ implementação da ESF, com consequente fortalecimento da APS tem colaborado significativamente para a melhoria dos indicadores de saúde no país, alterando o perfil de morbimortalidade da população e as hospitalizações desnecessárias. ${ }^{3}$

Apesar dos avanços decorridos desde a sua implantação, em 1994, ainda existem muitos desafios para a consolidação da ESF no Brasil. Um dos maiores problemas enfrentados pelos gestores de saúde é a fixação dos profissionais de saúde, especialmente os médicos ${ }^{4} \mathrm{~A}$ rotatividade desses profissionais pode comprometer a longitudinalidade do cuidado, a formação do vínculo das equipes com a população 
e, consequentemente, o alcance dos resultados esperados. ${ }^{5}$ Estudo realizado em Belo Horizonte verificou que o maior tempo de permanência do médico na equipe da APS foi associado a uma redução nas taxas de internação hospitalar. ${ }^{6}$ Ademais, a rotatividade produz custos elevados de várias naturezas, como de recrutamento e seleção para reposição de pessoal, de treinamento do recém-contratado e de desligamento. ${ }^{7}$

Com o objetivo de ampliar a mão de obra médica disponível no Brasil e solucionar a dificuldade de contratação de profissionais em algumas regiões, o Governo Federal lançou, em 2013, o "Programa Mais Médicos" (PMM) a partir da Medida Provisória $n^{\circ}$ 621, posteriormente convertida na Lei $n^{0} 12.871$. O programa foi estruturado a partir de três eixos de ação: (i) o investimento na melhoria da infraestrutura das redes de atenção à saúde; (ii) a ampliação da oferta de cursos e vagas em medicina e residências médicas, bem como a reestruturação da grade curricular com foco na valorização da atenção básica; e (iii) a implantação do "Projeto Mais Médicos" para o Brasil, que trata da provisão emergencial de médicos em áreas prioritárias para o sistema único de saúde (SUS), por meio de contrato temporário de 3 anos. ${ }^{8}$

Apesar do PMM estabelecer que médicos com registro no Brasil possuírem prioridade no preenchimento das vagas, houve vagas remanescentes após a etapa inicial. O segundo grupo na ordem de prioridade foram os médicos brasileiros formados no exterior sem registro médico no Brasil. Caso ainda haja vagas disponíveis, são, finalmente, convocados médicos estrangeiros com habilitação para exercício da medicina no exterior cujo diploma não tenha sido revalidado. ${ }^{8}$ Porém, em razão da escassez de médicos e falta de interesse de médicos brasileiros pelo programa, desde sua criação a maior parte da mão de obra médica foi composta por profissionais proveniente de Cuba, em uma parceria realizada pela Organização Pan-americana de Saúde (OPAS) entre os dois países. Porém, por mudanças governamentais ocorridas no Brasil, em novembro de 2018 houve ruptura do acordo, ${ }^{9}$ e o programa mais reconhecido, passou a ser mais atrativo por médicos com registro no país.

Alguns estudos brasileiros objetivaram identificar os fatores envolvidos na alta rotatividade dos médicos que compõem as equipes de ESF. Entre eles são citadas a grande carga de trabalho, ${ }^{10,11}$ condições de trabalho inadequadas, ${ }^{10,11}$ falta de médicos com perfil adequado para atuar na ESF ${ }^{12,13}$ e o alto índice de contratação de recém-formados, os quais saem da ESF para realização de residência médica. ${ }^{14}$

Reunir informações detalhadas acerca dos recursos humanos no setor de saúde é essencial para a gestão do sistema. ${ }^{15}$ A identificação do perfil dos profissionais faz-se necessária para a elaboração de estratégias que possam contribuir para a melhoria da qualidade dos serviços de saúde e das instituições de formação e aperfeiçoamento profissional, a fim de que se formem trabalhadores voltados para as reais necessidades da população.

O município de Ponta Grossa-PR, Brasil, localizado na mesorregião Centro Oriental Paranaense, tem uma população estimada de 348.043 habitantes. ${ }^{16} \mathrm{O}$ preenchimento das vagas para médicos na atenção primária mostra-se um desafio para os gestores locais. Segundo dados obtidos no endereço eletrônico da prefeitura, Ponta Grossa-PR foi o município brasileiro que proporcionalmente mais recebeu médicos cubanos através do PMM. Durante o acordo entre os dois países, $75 \%$ dos médicos da APS em Ponta Grossa-PR eram oriundos de Cuba. ${ }^{17}$

Nesse contexto, o objetivo deste estudo foi descrever o perfil sociodemográfico e os fatores de atração e saída dos médicos inseridos na estratégia de saúde da família (ESF) do município de Ponta Grossa-PR, Brasil.

\section{MÉTODOS}

Estudo transversal com abordagem quantitativa realizado nas unidades básicas de saúde do município de Ponta Grossa-PR, Brasil. 
No momento da pesquisa, segundo informações fornecidas pela secretaria municipal de saúde, 68 profissionais médicos atuavam nas equipes de ESF do município. Como critérios de inclusão foram considerados: ser médico integrante de equipe da ESF, assinar o termo de consentimento livre e esclarecido (TCLE) e não estar de afastamento médico ou férias no momento do estudo. Ao final, participaram da pesquisa 61 médicos, sendo o tamanho da amostra representativa, tendo em vista uma margem de erro de $5 \%(e=0,04)$.

Os dados foram obtidos a partir de questionário autoaplicado, entregue individualmente por uma única pesquisadora, para os médicos nas 42 unidades de saúde da família (USF) em que atuavam, nos meses de março e abril de 2019. O questionário foi baseado em outras pesquisas que avaliaram o perfil sociodemográfico e a motivação dos médicos na ESF, ${ }^{18,19}$ sendo composto por 35 questões relacionadas ao perfil sociodemográfico, formação, trajetória profissional e motivação pela ESF.

Os dados coletados foram tabulados no Excel 2013 (Microsoft) e realizou-se análise descritiva por meio de frequências absolutas ( $\mathrm{n}$ ) e relativas (\%).

O presente estudo foi autorizado pela secretaria municipal de saúde e aprovado pelo comitê de ética em pesquisa da Ponta Grossa-PR , através do parecer n³.183.547, em 6 de março de 2019.

\section{RESULTADOS}

Entre os 61 profissionais participantes, as mulheres representaram a maioria (57,4\%). A média de idade foi 34,9 anos (desvio padrão=11,0), com valores variando de 23 a 69 anos. Observou-se uma alta concentração de médicos na faixa etária abaixo dos 30 anos $(49,2 \%)$, não casados $(72,1 \%)$ e sem filhos $(67,2 \%)$. Renda per capita igual ou maior a 9 salários mínimos ( $\geq R \$ 8.982,00$ ) foi constatada em $81,4 \%$ dos entrevistados. Observase maior prevalência de profissionais não nascidos em Ponta Grossa-PR (82,0\%) (Tabela 1).

Tabela 1. Distribuição das características sociodemográficas dos médicos atuantes na ESF do município de Ponta GrossaPR , Brasil, 2019 ( $n=61)$.

\begin{tabular}{lcc}
\hline Variáveis & $\mathrm{n}$ & $\%$ \\
\hline Sexo & 35 & 57,4 \\
Feminino & 26 & 42,6 \\
Masculino & 30 & 49,2 \\
Faixa etária (anos) & 9 & 14,8 \\
$20-29$ & 16 & 26,2 \\
$30-39$ & 4 & 6,6 \\
$40-49$ & 2 & 3,3 \\
$50-59$ & & 72,1 \\
$\geq 60$ & 44 & 27,9 \\
Estado civil & 17 & 67,2 \\
Solteiro, viúvo ou divorciado & & 32,8 \\
Casado & 41 & 18,6 \\
Filhos & 20 & 81,4 \\
Não & & 18,0 \\
Sim & 11 & 31,2 \\
Renda per capita & 48 & 50,8 \\
Até 8 salários mínimos & & \\
L9 salários mínimos & 11 & \\
Local de Nascimento & 19 & \\
Ponta Grossa-PR & 31 & \\
Outra cidade do Paraná & & \\
Outro estado ou país & & \\
\hline
\end{tabular}


Verificou-se maior frequência de médicos que concluíram o ensino médio em instituições particulares $(59,0 \%)$, entretanto observou-se maior percentual de profissionais que concluíram a graduação em instituições públicas (52,5\%). Quanto à formação profissional, constatou-se que a maioria dos profissionais se graduaram após o ano de 2015 (60,7\%). Apenas 6,7\% dos entrevistados graduou-se em Ponta Grossa-PRe $61,7 \%$ concluiu sua graduação em outros estados brasileiros ou em outro país. O método de ensino mais utilizado na graduação foi o tradicional $(70,5 \%)$ e $65 \%$ dos médicos considerou que a preparação ofertada durante a graduação foi adequada para subsidiar sua atuação na ESF.

Sobre informações acerca de cursos de pós-graduação, observou-se que 1,6\% dos médicos possuíam residência em medicina de família de comunidade, 9,8\% tinha especialização em saúde da família e 32,8\% possui residência, especialidade e/ou pós-graduação em outras áreas. Para $37,7 \%$ dos profissionais, a ESF é a primeira experiência profissional na área médica (Tabela 2).

Tabela 2. Distribuição da formação profissional dos médicos atuantes na ESF do município de Ponta Grossa-PR , Brasil, $2019(n=61)$.

\begin{tabular}{|c|c|c|}
\hline Variável & $\mathbf{n}$ & $\%$ \\
\hline \multicolumn{3}{|l|}{ Ano de graduação } \\
\hline 2016 a 2018 & 37 & 60,7 \\
\hline$\leq 2015$ & 24 & 39,3 \\
\hline \multicolumn{3}{|l|}{ Cidade de graduação } \\
\hline Ponta Grossa-PR & 4 & 6,7 \\
\hline Outra cidade do Paraná & 19 & 31,6 \\
\hline Outro estado ou país & 37 & 61,7 \\
\hline \multicolumn{3}{|l|}{ Instituição de ensino médio } \\
\hline Pública & 25 & 41,0 \\
\hline Privada & 36 & 59,0 \\
\hline \multicolumn{3}{|l|}{ Instituição de graduação } \\
\hline Pública & 32 & 52,5 \\
\hline Privada & 29 & 47,5 \\
\hline \multicolumn{3}{|c|}{ Método de ensino da graduação } \\
\hline Tradicional & 43 & 70,5 \\
\hline Não tradicional (PBL/misto) & 18 & 29,5 \\
\hline \multicolumn{3}{|l|}{ Residência em MFC ${ }^{1}$} \\
\hline Sim & 1 & 1,6 \\
\hline Não & 60 & 98,4 \\
\hline \multicolumn{3}{|l|}{ Especialização em SF ${ }^{2}$} \\
\hline Sim & 6 & 9,8 \\
\hline Não & 55 & 90,2 \\
\hline \multicolumn{3}{|c|}{ Outra especialidade/pós-graduação } \\
\hline Sim & 20 & 32,8 \\
\hline Não & 41 & 67,2 \\
\hline \multicolumn{3}{|l|}{ Outro emprego antes da ESF } \\
\hline $\operatorname{Sim}$ & 38 & 62,3 \\
\hline $\begin{array}{l}\text { Não teve outro emprego } \\
\text { Graduação foi suficiente pa }\end{array}$ & 23 & 37,7 \\
\hline $\operatorname{Sim}$ & 39 & 65,0 \\
\hline Não & 21 & 35,0 \\
\hline
\end{tabular}

Legendas: ${ }^{1}$ Medicina de família e comunidade; ${ }^{2}$ Saúde da família. 
Quanto à trajetória na ESF (Tabela 3), constatou-se alta concentração de médicos que trabalhavam na ESF há menos de 6 meses (59\%) e de profissionais que ingressaram no serviço através do "Programa Mais Médicos" (82\%). Segundo análise da variável "tempo que pretende continuar trabalhando na ESF", foi possível observar que $77,1 \%$ dos médicos pretendia sair em um prazo de 5 anos, sendo que a maioria $(45,9 \%)$ respondeu que poderia sair a qualquer momento ou em um prazo de até 2 anos.

Apenas 29,5\% relatou outros vínculos empregatícios e 73,8\% afirmou trabalhar até 40 horas semanais. Questionados quanto à remuneração na ESF, 52,5\% consideraram "adequada", 21,3\% disseram ser "muito boa" e apenas $26,2 \%$ consideraram "ruim" ou "regular". Os médicos foram unânimes em responder que a atenção primária à saúde é um ponto chave para a melhoria do serviço de saúde no Brasil.

Dos 48 médicos $(78,7 \%)$ que afirmaram pretender fazer alguma residência médica, somente 14,6\% pretende fazer residência em medicina de família e comunidade (MFC).

Tabela 3. Distribuição dos vínculos empregatícios dos médicos atuantes na ESF do município de Ponta Grossa-PR , Brasil, $2019(n=61)$.

\begin{tabular}{|c|c|c|}
\hline Variáveis & $\mathbf{N}$ & $\%$ \\
\hline \multicolumn{3}{|l|}{ Tempo de atuação na ESF } \\
\hline Menos de 6 meses & 36 & 59,0 \\
\hline 6 meses a 2 anos & 9 & 14,8 \\
\hline Entre 2 e 5 anos & 1 & 1,6 \\
\hline Mais de 5 anos & 15 & 24,6 \\
\hline \multicolumn{3}{|l|}{ Pretende continuar na ESF } \\
\hline No máximo 2 anos & 20 & 32,8 \\
\hline Entre 2 e 5 anos & 19 & 31,2 \\
\hline Mais de 5 anos & 3 & 4,9 \\
\hline Não pretende sair & 11 & 18,0 \\
\hline Não pretende continuar & 8 & 13,1 \\
\hline \multicolumn{3}{|l|}{ Forma de ingresso na ESF } \\
\hline Concurso público & 11 & 18,0 \\
\hline "Programa Mais Médicos" & 50 & 82,0 \\
\hline \multicolumn{3}{|l|}{ Outro vínculo empregatício } \\
\hline Sim & 18 & 29,5 \\
\hline Não & 40 & 65,6 \\
\hline Não respondeu & 3 & 4,9 \\
\hline \multicolumn{3}{|c|}{ Carga horária total de trabalho } \\
\hline Até $40 \mathrm{~h} /$ semana & 45 & 73,8 \\
\hline Mais de $40 \mathrm{~h} /$ semana & 16 & 26,2 \\
\hline Variáveis & $\mathrm{N}$ & $\%$ \\
\hline \multicolumn{3}{|c|}{ Considera a remuneração na ESF } \\
\hline Muito boa & 13 & 21,3 \\
\hline Adequada & 32 & 52,5 \\
\hline Regular & 11 & 18,0 \\
\hline Ruim & 5 & 8,2 \\
\hline
\end{tabular}

O principal motivo apontado pelos médicos para a sua escolha pela ESF foi a identificação com o trabalho. Dos que não pretendiam continuar trabalhando na ESF ou pretendiam sair em até 2 anos, 67,9\% mencionaram que estão aguardando aprovação na residência médica desejada (Figura 1). 


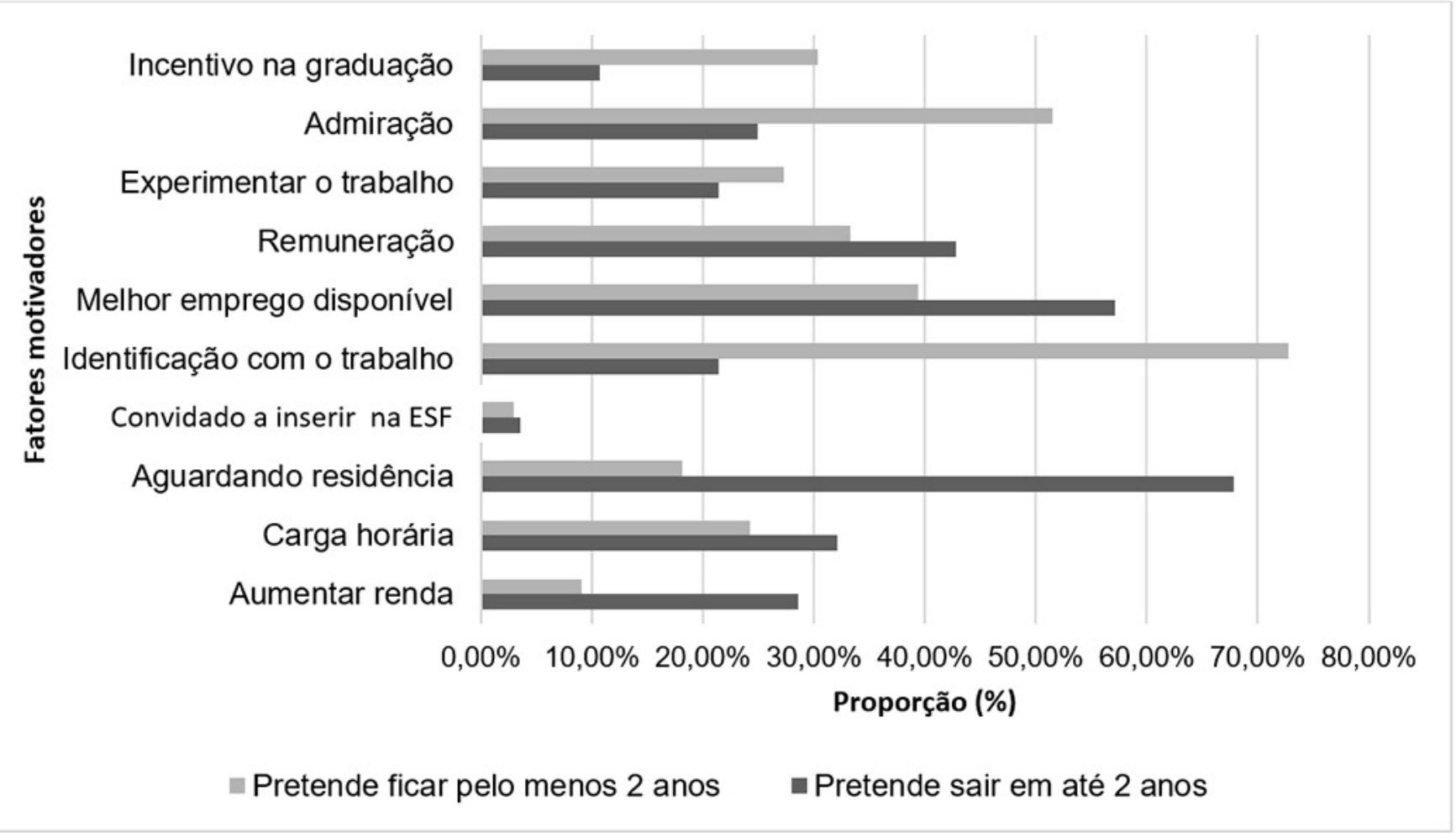

Figura 1. Distribuição dos fatores motivadores para inserção na ESF, segundo pretensão de permanecer ou não no mínimo 2 anos, por médicos atuantes na ESF no município de Ponta Grossa-PR, Brasil, 2019.

Legenda: A soma das porcentagens excede $100 \%$, porque poderia ser assinalado mais de uma opção.

Questionados quanto aos fatores que poderiam levá-los a deixar a ESF, 68,9\% responderam residência médica (Figura 2). Os outros fatores mais relevantes foram "outro emprego com remuneração melhor" $(27,9 \%)$, mudança no tipo de vínculo $(19,7 \%)$ e outro emprego na cidade de origem $(16,3 \%)$.

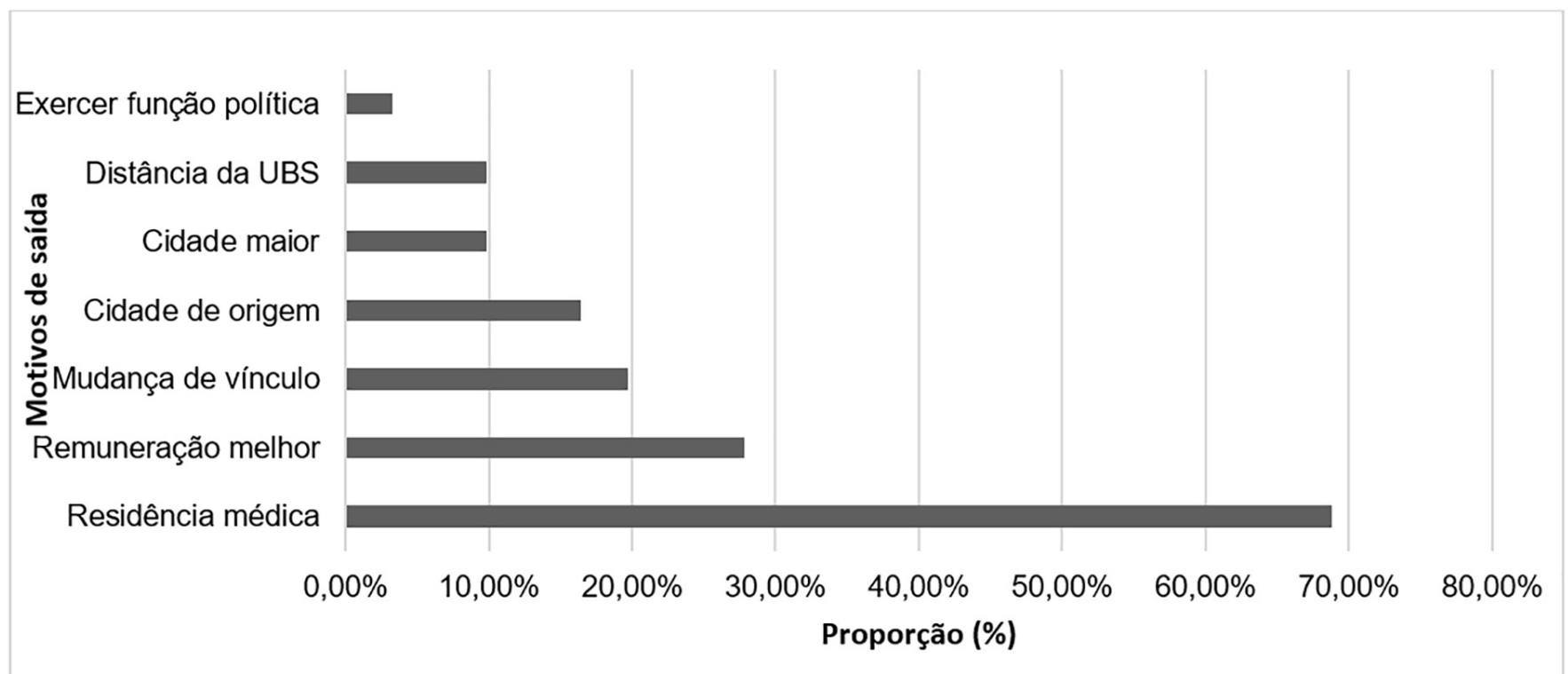

Figura 2. Distribuição dos fatores que poderiam levar à saída dos médicos da ESF, Ponta Grossa-PR, Brasil, 2019.

Legenda: A soma das porcentagens excede $100 \%$, porque poderia ser assinalado mais de uma opção. 
Em relação aos fatores que levam à desmotivação na ESF, o excesso de demanda e de burocracia foram os mais citados com frequência similar (65,6\%). Remuneração foi considerada um fator de desmotivação por apenas $18 \%$ dos médicos (Figura 3). Essa questão permitia uma resposta aberta onde o profissional poderia relatar outros fatores que não estavam entre os listados. Problemas com a gestão e dificuldade na realização de exames complementares apareceram entre os principais, ambos citados por $6,6 \%$ dos entrevistados.

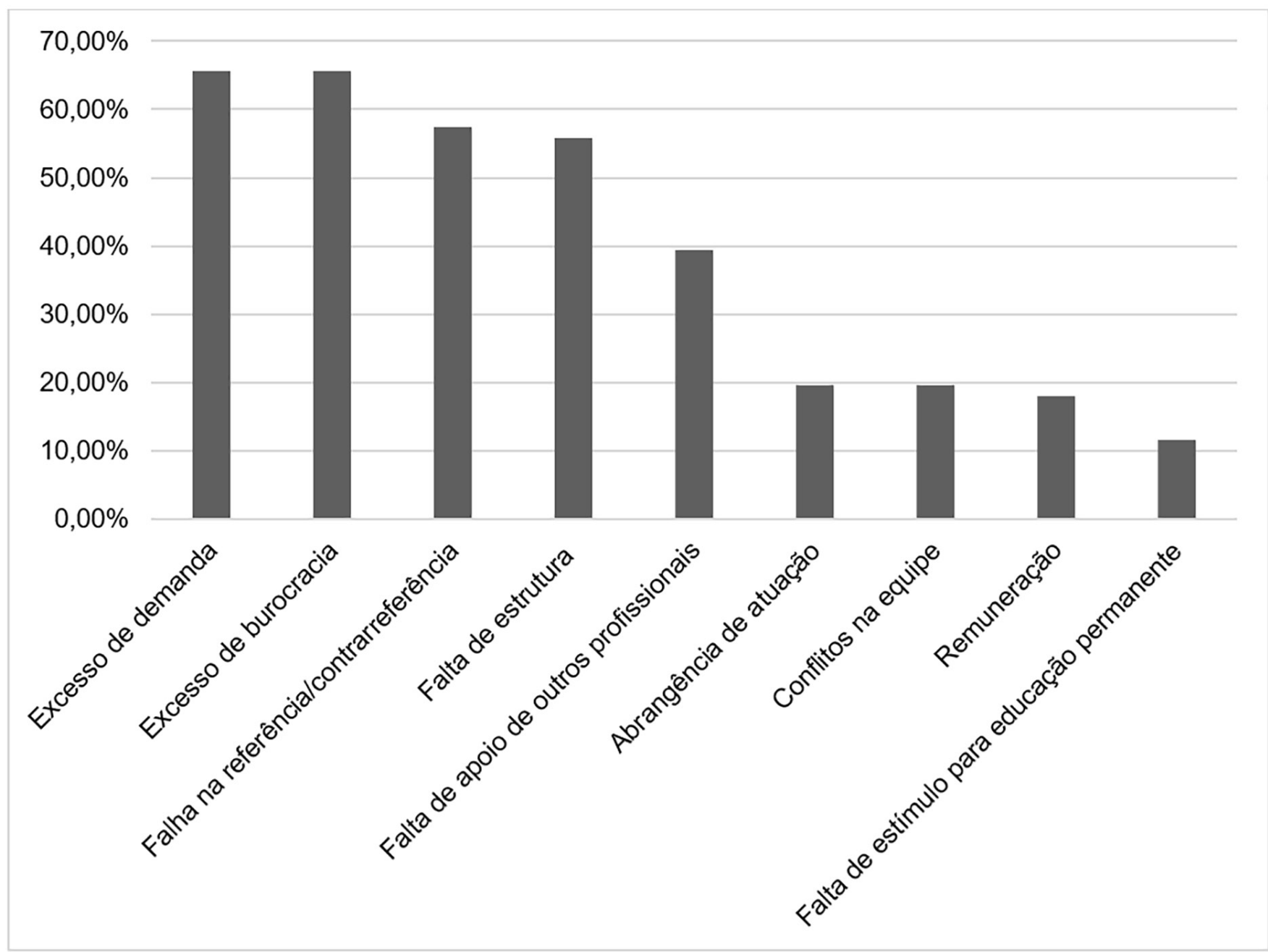

Figura 3. Distribuição dos fatores de desmotivação na ESF por médicos atuantes na ESF no município de Ponta Grossa-PR,, Brasil, 2019. Legenda: A soma das porcentagens excede $100 \%$, porque poderia ser assinalado mais de uma opção

\section{DISCUSSÃO}

A predominância das mulheres atuando na ESF coincide com vários estudos brasileiros semelhantes ${ }^{19-22}$ e reflete uma tendência nacional à feminilização da área da saúde. ${ }^{23,24}$ Alguns autores mostram que as mulheres têm maior facilidade no desenvolvimento da relação médico-paciente e na abordagem de assuntos emocionais, ${ }^{25}$ além de serem mais propensas a realizarem procedimentos de prevenção, ${ }^{26}$ habilidades importantes no contexto da APS.

A média de idade (34,9 anos) foi inferior à média nacional encontrada em um estudo recente sobre a demografia médica no Brasil $^{27}$ (45,4 anos). O predomínio da faixa etária até 29 anos (49,2\%), bem como o alto número de profissionais formados após 2015 (60,7\%) pode ser justificada pelo grande número de 
vagas ofertadas na ESF, para as quais exige-se apenas nível de graduação, diferentemente de outras áreas de atuação da medicina, às quais comumente exige-se uma maior qualificação. $O$ aumento expressivo do número de vagas em cursos de medicina que, em pouco menos de cinco décadas, aumentou 665,8\% $(7,7$ vezes $)^{27}$ também pode explicar a alta proporção de médicos jovens. Além disso, a nova Diretriz Curricular dos Cursos de Graduação em Medicina, ${ }^{28}$ aprovada em 2014, possibilitou aos acadêmicos um maior contato com a atenção primária em saúde e pode explicar em parte a escolha dos médicos jovens pela ESF.

A alta prevalência encontrada de médicos não casados $(72,1 \%)$, sem filhos $(67,2 \%)$ e que não nasceram (82\%) ou não se graduaram em Ponta Grossa-PR, $(93,3 \%)$ pode predispor à rotatividade, uma vez que a literatura aponta que a presença de cônjuge e filhos, ${ }^{29}$ e o trabalho no município de origem são fatores de retenção desses profissionais. ${ }^{27,29}$

A renda per capita majoritariamente igual ou superior a 9 salários mínimos $(81,4 \%)$ foi similar à verificada por Guarda et al., em 2012, ${ }^{18}$ em estudo realizado com os médicos da ESF na região metropolitana do Recife $(86,9 \%)$.

O predomínio de médicos que cursaram o ensino médio em instituições particulares (59\%) coincide com dados encontrados em pesquisa recente com 4.601 profissionais formados de 2014 a 2015 (79,1\%). ${ }^{27}$ Verificou-se equilíbrio na distribuição de profissionais graduados em instituições de nível superior públicas $(52,5 \%)$ e privadas $(47,5 \%)$, sugerindo que a modalidade da instituição não exerceu grande influência na escolha pela ESF.

O alto índice de profissionais sem especialidade ou pós-graduação é esperado, visto a predominância de recém-formados inseridos na APS. No entanto, a porcentagem pequena de médicos com especialização em medicina de família e comunidade ou saúde da família (1,6\%), bem como a baixa intenção em realizar residência em MFC $(11,5 \%)$ reflete o desinteresse dos profissionais pela área. Apesar do incentivo governamental a novos programas e vagas de residência, a especialidade, considerada estratégica para o SUS, em 2017, contava com apenas 5.486 médicos (1,4\% do total de médicos especialistas no Brasil) com alto número de vagas da residência em MFC continuam ociosas. ${ }^{27}$

Quanto à trajetória profissional, a maioria dos profissionais teve outros empregos como médicos antes do ingresso na ESF, em concordância com dados encontrados na literatura. ${ }^{19,22}$

Apesar da maioria dos entrevistados ter considerado que a abordagem sobre APS na graduação foi suficiente para subsidiar sua atuação na ESF, um percentual significativo (35\%) considerou sua formação inadequada, sugerindo necessidade de aprimoramento dos cursos de medicina nesse quesito, a fim de cumprir seu objetivo, estipulado nas diretrizes curriculares nacionais, de formar médicos generalistas na perspectiva de integralidade da assistência, considerando as necessidades de saúde mais frequentemente identificadas. ${ }^{28}$ Vale destacar aqui que o município de Ponta Grossa-PR, conta com uma instituição de ensino superior (IES) pública com o curso de medicina, com mais de 10 anos de implantação, e a aproximação entre ensino-serviço deve ser fortalecida e pode favorecer ambos setores (saúde e educação).

Nesse estudo, o elevado número de contratações realizadas através do "Programa Mais Médicos", assim como a alta proporção de profissionais que trabalham na ESF há menos de 6 meses, deve-se ao fato de até recentemente (novembro de 2018) o município possuir $75 \%$ de suas equipes de ESF preenchidas por trabalhadores cubanos. ${ }^{17}$ Após a ruptura, em novembro de 2018, do acordo entre Brasil e Cuba, ${ }^{9}$ esses profissionais se retiraram do programa, gerando a necessidade de novas contratações 
para suprir a demanda. Nesse sentido, o município parece ter se pautado na absorção da provisão de profissionais, mas vale destacar que o programa tem caráter provisório e emergencial, especialmente em áreas com vazios assistenciais. No entanto, o município de grande porte fica localizado em uma região estratégica de desenvolvimento do estado XXX, na região Sul do Brasil. Nesse sentido, precisa adequar-se para absorver as outras etapas do PMM, inclusive qualificar a formação de médicos para a APS, como o pró-residência. Se essa e/ou outras medidas de fixação de médicos na região não forem implementadas de forma estratégica, o município apresenta uma dependência do governo federal, e não terão médicos fixados a região e, dificilmente, haverá investimento na qualificação da APS.

O número de contratações mediante concurso público (18\%) foi consideravelmente inferior ao encontrado em estudos semelhantes, os quais apontaram que a maioria dos médicos ingressou via concurso. ${ }^{18,19,22}$ A precarização da forma de contratação associa-se a uma menor permanência, ${ }^{29}$ contribuindo para a expressiva rotatividade médica encontrada no município, que possui apenas $26,2 \%$ de seus profissionais trabalhando na ESF há mais de 2 anos.

Destaca-se ainda que o município não tem sido atrativo para a fixação de profissionais formados na IES localizada no município, e isso pode se dar por diversos fatores, como salariais ou plano de carreira, uma vez que tem sido realizado concursos com frequência. Em relação à universidade, ela precisa repensar a lógica de formação de recurso humano para responder as necessidades locais e desenvolvimento locorregional, mesmo que haja acadêmicos oriundos de outras regiões do estado ou país. Nesse caso, uma formação médica generalista voltada para a APS, especialmente em medicina de família e comunidade, em nível de graduação e pós-graduação, e estímulos municipais para a fixação de profissionais deve estar bem evidente.

Quanto à pretensão de continuar trabalhando na ESF, verificou-se que o município pode perder $45,9 \%$ da mão de obra médica nas equipes em até 2 anos. O principal motivo apontado para a saída foi a realização de residência médica, dados esses corroborado por outros estudos. ${ }^{18,29}$ Inicialmente, uma forma de incentivo ao trabalho na APS foi por meio da bonificação em processos seletivos em diversos programas de residência médica e de programas específicos, como o "Programa de Valorização do Profissional da Atenção Básica" (PROVAB), ${ }^{30}$ mas não existiu a continuidade. Sendo assim, a APS ainda continua sendo vista como um lugar de transição na carreira médica, como uma etapa entre a graduação e a especialidade ou sistema privado. Nogueira (2006) ${ }^{31}$ ressalta que os médicos da ESF não se sentem em uma especialidade efetiva, plenamente reconhecida e valorizada.

O quantitativo de médicos que não possuem outros vínculos empregatícios além da ESF foi superior ao encontrado em outros estudos, ${ }^{18,22}$ o que pode ser um indicador positivo, visto que o excesso de trabalho pode acarretar riscos e prejuízos para a qualidade do serviço, bem como à saúde do profissional. ${ }^{32}$ No entanto, pode refletir a dificuldade de profissionais médicos generalistas em se inserir no mercado de trabalho liberal, ou ainda, alcançar uma renda onde conseguem ter uma estabilidade financeira, com menor tempo de trabalho, para dedicar-se aos estudos para a residência pretendida. Isso foi visto no estudo quando o grupo de profissionais que pretendia sair em até 2 anos do serviço público, a principal motivação foi o aguardo da residência médica (67,9\%).

A identificação com o trabalho foi o fator que mais motivou os médicos a ingressarem na ESF, em conformidade com a literatura. ${ }^{22,29}$ Rodrigues, Silva e Rocha $(2008)^{29}$, em estudo realizado em Minas 
Gerais, verificaram que, dentre os médicos que ingressam no serviço público aguardando a oportunidade de fazer residência, apenas $10 \%$ permanecem por pelo menos 5 anos, concluindo que o motivo que levou o profissional a trabalhar na ESF também parece estar associado à sua permanência.

A remuneração, frequentemente apontada como fator crítico na atração de profissionais para a APS, foi considerada regular ou ruim por apenas $26,2 \%$ dos entrevistados, e apenas $18 \%$ considerou a questão salarial como um fator de desmotivação. O fato do público respondente na pesquisa ser majoritariamente de recém-formados, possivelmente recebendo os primeiros salários e sem um gasto fixo e familiar alto, torna-se um valor suficientemente atrativo. O mesmo não ocorre para profissionais com maior tempo de carreira, oriundos de outras cidades, estados ou países, e precisam manter-se em uma nova cidade de trabalho, o que pode dificultar a permanência no serviço público ou a busca de outros serviços mais bem remunerados posteriormente.

Dentre os fatores citados como geradores de desmotivação na ESF, destaca-se o excesso de demanda, que pode ser em parte justificado pela falta de médicos encontrada em diversas equipes, fazendo com que muitos profissionais precisem absorver a demanda de outros territórios além da sua população adscrita. Soma-se a isso, o excesso de processos burocráticos, que leva a uma redução ainda maior no tempo efetivo que o médico possui para dedicar-se aos cuidados em saúde. Outro ponto consideravelmente citado foram as falhas no processo de referência e contra referência, resultado semelhante ao encontrado em outros estudos, ${ }^{19,33}$ indicando falta de articulação na rede de atenção à saúde, mesmo sendo um município de grande porte e com equipamentos de diferentes níveis de atenção no próprio município.

Um estudo apontou que as condições de trabalho são o principal determinante para fixação em um emprego ou cidade (84\%). ${ }^{27}$ Rodrigues et al. (2010) ${ }^{29}$ encontraram associação entre o nível de satisfação com o trabalho e a permanência do médico na ESF. Logo, os fatores de desmotivação refletem prejuízo na assistência prestada como também na escassez e rotatividade da mão-de-obra médica, sendo prejudicial para todos os agentes envolvidos no setor de saúde.

A limitação do estudo esta relacionado ao seu recorte transversal, o qual não se pode inferir causalidade. Além disso, apesar do tamanho amostral ser representativo, conta com uma amostra pequena de profissionais. Apesar de poder dimensionar a realidade de outros municípios brasileiros, a realidade deste município brasileiro, localizado no interior do Paraná, apresenta singularidades na realização ou adesão de políticas públicas de saúde em nível municipal, estadual e nacional, na capacidade de formação de recursos humanos, porte do município e cobertura de APS, dentre outros fatores.

Os resultados desse estudo podem contribuir para um melhor entendimento dos motivos que levam à alta rotatividade dos médicos da APS no município, bem como em outras localidades. Devido ao número limitado de médicos entrevistados, considerando-se o universo da ESF, cabe ressaltar a necessidade de outros estudos que analisem as possíveis associações entre o perfil sociodemográfico e a rotatividade desses profissionais.

\section{CONCLUSÃO}

O predomínio de profissionais com baixo tempo de trabalho na APS do município oriundos do "Programa Mais Médicos" ressalta a necessidade de um plano de substituição desse tipo de vínculo, que 
é baseado em contratos temporários e relações trabalhistas precárias. Apesar de necessário, o PMM deve ser visto como uma medida emergencial e provisória.

A elaboração de estratégias de valorização da carreira médica na atenção primária à saúde pode ajudar a tornar o trabalho na ESF mais atrativo para os profissionais. Nesse aspecto, uma solução poderia ser a criação de um plano de carreira considerando o tempo de serviço e o nível de especialização.

A oferta de melhores condições de trabalho também parece ser um ponto chave para a fixação dos trabalhadores. O excesso de demanda, destacado por muitos entrevistados, poderia ser minimizado com investimentos na capacitação da equipe, reduzindo assim a carga de trabalho sobre o médico.

Faz-se necessária, também, a ampliação da oferta de serviços especializados, a fim de facilitar o processo de referência e contrarreferência. A implementação de um sistema de prontuários eletrônicos integrados poderia ser útil nesse contexto, o que possibilitaria a troca eficiente de informações entre os diversos pontos da rede de atenção.

Nota-se, porém, que a rotatividade médica é um assunto complexo, o qual envolve questões políticas, pessoais e sociais. Nesse sentido uma maior articulação entre os setores de educação e saúde pode estabelecer medidas mais assertivas para reduzir os impactos do baixo vínculo dos profissionais médicos na APS.

\section{Contribuições dos autores}

Concepção e/ou delineamento do estudo: LSN, EVM.

Aquisição, análise ou interpretação dos dados: LSN, EVM, MFS.

Redação preliminar: LSN.

Revisão crítica da versão preliminar: EVM, MFS.

Todos os autores aprovaram a versão final e concordaram com prestar contas sobre todos os aspectos do trabalho.

\section{Conflito de interesses}

Os autores informam não haver conflitos de interesse.

\section{REFERÊNCIAS}

1. Starfield B. Atenção primária: equilíbrio entre necessidades de saúde, serviços e tecnologia. Brasília (DF): UNESCO/Ministério da Saúde; 2002.

2. Ministério da Saúde (BR). Portaria no 2.488, de 21 de outubro de 2011. Aprova a Política Nacional de Atenção Básica, estabelecendo a revisão de diretrizes e normas para a organização da Atenção Básica para a Estratégia Saúde da Família (ESF) e o Programa Agentes Comunitários de Saúde (PACS). Diário Oficial da União, Brasília (DF), 21 out 2006; Seção 1: 1.

3. Paim J, Travassos C, Almeida C, Bahia L, Macinko J. O sistema de saúde brasileiro: história, avanços e desafios. Lancet. 2011 Mai;377(9779):1778-97. DOI: https://doi.org/10.1016/S0140-6736(11)60054-8

4. Escorel S, Giovanella L, Mendonça MHM, Senna MCM. O programa de saúde da família e a construção de um novo modelo para a atenção básica no Brasil. Pan Am J Public Health. 2007; [citado 2019 Mai 30]; 21(2):164-76. Disponível em: https://scielosp.org/pdf/ rpsp/2007.v21n2-3/164-176/pt DOI: https://doi.org/10.1590/S1020-49892007000200011

5. Reddy A, Pollack CE, Asch DA, Canamucio A, Werner RM. The effect of primary care provider turnover on patient experience of care and ambulatory quality of care. JAMA Intern Med. 2015 Jul;175(7):1157-62. DOI: https://doi.org/10.1001/jamainternmed.2015.1853 PMID: 25985320 
Perfil sociodemográfico e fatores de atração e saída dos médicos

6. Mendonça CS, Harzheim E, Duncan BB, Nunes LN, Leyh W. Trends in hospitalizations for primary care sensitive conditions following the implementation of Family Health Teams in Belo Horizonte, Brazil. Health Policy Plan. 2012 Jul;27(4):348-55. DOI: https://doi.org/10.1093/ heapol/czr043

7. Chiavenato I. Recursos humanos: o capital humano das organizações. 9aㅡ ed. Rio de Janeiro: Elsevier Campus; 2009.

8. Portaria Interministerial no 1.369, de 8 de julho de 2013 (BR). Dispõe sobre a implementação do Projeto Mais Médicos para o Brasil. Diário Oficial da União, Brasília (DF), 8 jul 2013; Seção 3: 204.

9. Ferreira L, Souza A. Ministério da Saúde de Cuba anuncia que país sairá do programa Mais Médicos. O Globo [Internet]. 2018 Nov; [acesso em 2019 Mai 26]. Disponível em: https://g1.globo.com/mundo/noticia/2018/11/14/cuba-decide-deixar-programa-mais-medicosno-brasil.ghtml

10. Ney MS, Rodrigues PHA. Fatores críticos para a fixação do médico na Estratégia Saúde da Família. Physis. 2012;22(4):1293-311. DOI: http://dx.doi.org/10.1590/S0103-73312012000400003

11. Capozzolo AA. No olho do furacão: trabalho médico e o Programa de Saúde da Família [tese na Internet]. Campinas (SP): Universidade Estadual de Campinas (Unicamp) - Faculdade de Ciências Médicas em Saúde Pública; 2018; [acesso em 2018 Set 30]. Disponível em: http://bvsms.saude.gov.br/bvs/ct/pdf/angela\%20aparecida_tese.pdf

12. Ministério da Saúde (BR). Secretaria de Políticas da Saúde. Perfil dos médicos e enfermeiros do Programa de Saúde da Família. Brasília (DF): Ministério da Saúde; 2000.

13. Campos CV, Malik AM. Satisfação no trabalho e rotatividade dos médicos do Programa de Saúde da Família. Rev Adm Pública. 2008 Abr;42(2):347-68. DOI: http://dx.doi.org/10.1590/S0034-7612200800020000714. Pierantoni CR. As reformas de Estado, da saúde e recursos humanos: limites e possibilidades. Ciênc Saúde Colet. 2001;6(2):341-60. DOI: http://dx.doi.org/10.1590/S141381232001000200006

15. Arditi C, Burnand B. Démographie médicale: indicateurs et observatoires [Internet]. Lausanne: Institut Universitaire de Médecine Sociale et Préventive; 2014; [citado 2019 Mai 27]; 236:1-84. Disponível em: https://www.iumsp.ch/fr/rds/236

16. Instituto Brasileiro de Geografia e Estatística (IBGE). Estimativa populacional 2018 IBGE - Ponta Grossa [Internet]. Brasília (DF): IBGE; 2018; [acesso em 2019 Fev 04]. Disponível em: https://cidades.ibge.gov.br/brasil/pr/ponta-grossa/panorama

17. Ramos R. Mais Médicos: Ponta Grossa recebe mais 14 médicos cubanos para atendimento na Atenção Primária [Internet]. Ponta Grossa (PR): Prefeitura de Ponta Grossa;2017; [acesso em 2018 Set 24]. Disponível em: http://www.pontagrossa.pr.gov.br/node/36368

18. Guarda FRB, Silva RN, Tavares RAW. Perfil sociodemográfico dos médicos que compõem equipes de saúde da família na Região Metropolitana do Recife, Estado de Pernambuco, Brasil. Rev Pan-Amaz Saude. 2012 Jun;3(2):17-24. DOI: http://dx.doi.org/10.5123/ S2176-62232012000200003

19. Damno HS, Moriyama MC, Pícoli RP, Sartori BG, Asato SMM, Carbalho GC, et al. Perfil profissional dos médicos atuantes na estratégia Saúde da Família no Município de Campo Grande-MS. Encontro Rev Psicol [Internet]. 2013; [citado 2018 Out 29]; 16(25):125-37. Disponível em http://revista.pgsskroton.com.br/index.php/renc/article/download/2437/2335

20. Costa SM, Prado MCM, Andrade TN, Silva Junior WS, Gomes Filho ZC, Rodrigues CAQ. Perfil do profissional de nível superior nas equipes da Estratégia Saúde da Família em Montes Claros, Minas Gerais, Brasil. Rev Bras Med Fam Comunidade. 2013 Abr;8(27):90-6. DOI: https://doi.org/10.5712/rbmfc8(27)530

21. Lima EFA, Sousa AI, Primo CC, Leite FMC, Souza MHN, Maciel ELN. Perfil Socioprofissional de trabalhadores de equipes saúde da família. Rev Enferm UERJ. 2016;24(1):9405. DOI: http://dx.doi.org/10.12957/reuerj.2016.9405

22. Mendes CLA. Perfil do profissional médico na Estratégia de Saúde da Família no município do Rio de Janeiro: um modelo em transição [dissertação na Internet]. Rio de Janeiro (RJ): Escola Nacional de Saúde Pública Sérgio Arouca (ENSP); 2015; [acesso em 2019 Mai 27]. Disponível em: http://bases.bireme.br/cgi-bin/wxislind.exe/iah/online/?lsisScript=iah/iah.xis\&src=google\&base=LILACS\&lang=p\&n extAction=Ink\&exprSearch=782511\&indexSearch=ID

23. Wermelinger M, Machado MH, Tavares MFL, Oliveira ES, Moyses NN, Ferraz W. A feminilização do mercado de trabalho em saúde no Brasil. Divulg Saúde Debate [Internet]. 2010; [citado 2019 Mai 30]; 45:54-70. Disponível em: http://bases.bireme.br/cgi-bin/wxislind.exe/ iah/online/?IsisScript=iah/iah. xis\&src=google\&base=LILACS\&lang=p\&nextAction=Ink\&exprSearch=565543\&indexSearch=ID

24. Scheffer MC, Cassenote AJF. A feminização da medicina no Brasil. Rev Bioét. 2013 Ago;21(2):268-77. DOI: http://dx.doi.org/10.1590/ S1983-80422013000200010

25. Roter DL, Hall JA, Aoki Y. Physician gender effects in medical communication: a meta-analytic review. JAMA. 2002 Ago;288(6):756-64. DOI: http://dx.doi.org/10.1001/jama.288.6.756 
26. Franks P, Bertakis KD. Physician gender, patient gender, and primary care. J Womens Health. 2003 Jul;12(1):73-80. DOI: http://dx.doi. org/10.1089/154099903321154167

27. Scheffer MC, Cassenote A, Guilloux AGA, Miotto BA, Mainardi GM; Conselho Federal de Medicina (CFM), et al. Demografia médica no Brasil 2018 [Internet]. São Paulo (SP): FMUSP/CFM/Cresmesp; 2018; [acesso em 2019 Mai 30]. Disponível em https://jornal.usp.br/ wp-content/uploads/DemografiaMedica2018.pdf

28. Ministério da Educação (BR). Conselho Nacional de Educação. Câmara de Educação Superior. Resolução no 3, de 20 de junho de 2014. Diretrizes Curriculares Nacionais do Curso de Graduação em Medicina e outras providências. Brasília (DF): Ministério da Educação; 2014.

29. Rodrigues RB, Silva NC, Rocha TAH. Atração e retenção do profissional médico e os desafios para a Estratégia Saúde da Família. In: Encontro da Associação Nacional de Pós-graduação e Pesquisa em Administração (ANPAD) [Internet]. Rio de Janeiro, Rio de Janeiro, Brasil, 25-29 set 2010. Rio de Janeiro (RJ): ANPAD; 2010; [acesso em 2019 Mai 30]; p. 1-17. Disponível em: http://www.anpad.org.br/ admin/pdf/gpr2161.pdf

30. Ministério da Saúde (BR). Gabinete do Ministro. Portaria Interministerial no 2.087, de 1 de setembro de 2011. Institui o Programa de Valorização do Profissional da Atenção Básica [Internet]. Brasília (DF): Ministério da Saúde; 2011; [acesso em 2019 Mai 27]. Disponível em: http://bvsms.saude.gov.br/bvs/saudelegis/gm/2011/pri2087_01_09_2011.html

31. Nogueira RP. A questão do médico em saúde da família. Rev Desafios Desenvolvimento [Internet]. 2006; [citado 2019 Mai 30]; 28(3): Disponível em: http://www.ipea.gov.br/desafios/index.php?option=com_content\&view=article\&id=85:catid=28\&ltemid=23

32. Murofuse NT, Rizzotto MLF, Muzzolon ABF, Nicola AL. Diagnóstico da situação dos trabalhadores em saúde e o processo de formação no polo regional de educação permanente em saúde. Rev Latino-Am Enferm. 2009 Jun;17(3):314-20. DOI: http://dx.doi.org/10.1590/ S0104-11692009000300006 DOI: https://doi.org/10.1590/S0104-11692009000300006

33. Gonçalves RJ, Soares RA, Troll T, Cyrino EG. Ser médico no PSF: formação acadêmica, perspectivas e trabalho cotidiano. Rev Bras Educ Med. 2009 Set;33(3):382-92. DOI: http://dx.doi.org/10.1590/S0100-55022009000300009 
